\section{Práticas sociais de medicalização \& humanização no cuidado de mulheres na gestação}

\author{
Social practices in the medicalization and \\ humanization of prenatal care
}

\section{Prácticas sociales de medicalización y humanización en el cuidado de mujeres durante el embarazo}

Cristine Maria Warmling 1

Ananyr Porto Fajardo 2

Dagmar Estermann Meyer 3

Cristophe Bedos 4

\title{
Resumo
}

O objetivo principal do trabalho é analisar como discursos de medicalização \& humanização se (re)articulam na atenção primária em saúde e configuram o cuidado pré-natal de mulheres grávidas realizado por equipes de saúde da família. Trata-se de um estudo de caso do tipo único e integrado, com múltiplas unidades de análises e abordagem qualitativa. Foram realizados 17 grupos focais e ouvidos 47 trabalhadores (14 médicos, 19 enfermeiros e 14 cirurgiões-dentistas) que compunham 17 equipes de saúde da família em 16 municípios no Sul do Brasil. O material empírico foi analisado na perspectiva da análise do discurso foucaultiana. As equipes de saúde da família, praticantes da medicina generalista, relataram dificuldades para realizar 0 cuidado pré-natal das mulheres gestantes, evocando e fortalecendo o discurso da medicalização obstétrica que sua prática deveria enfraquecer. O discurso oficialmente adotado pela humanização, privilegiado no modelo generalista de atenção às mulheres gestantes, segue funcionando como discurso complementar ao da medicalização e da especialização, que prevalece nas práticas relatadas. A ênfase na atenção humanizada à mulher na gestação interfere nas fronteiras dos territórios profissionais e pressupõe renegociação de competências. Esforços de colaboração empreendidos entre as equipes de saúde da família e obstetras não apresentam muito sucesso.

Medicalização; Parto Humanizado; Gestantes

\author{
Correspondência \\ C. M. Warmling \\ Rua Dona Leonor 194, apto. 1407, Porto Alegre, RS \\ 90420-180, Brasil. \\ crismwarm@gmail.com \\ 1 Faculdade de Odontologia, Universidade Federal do Rio \\ Grande do Sul, Porto Alegre, Brasil. \\ 2 Grupo Hospitalar Conceição, Porto Alegre, Brasil. \\ 3 Faculdade de Educação, Universidade Federal do Rio Grande \\ do Sul, Porto Alegres, Brasil. \\ ${ }_{4}^{4}$ Faculty of Dentistry, McGill University, Montreal, Canada.
}




\section{Introdução}

A medicalização do corpo grávido, do parto e do nascimento tem se configurado como um processo em expansão no Brasil. De forma bem evidente é um processo expresso pelas altas taxas de cesáreas (que estão entre as mais altas do mundo), e que são aceitas como "naturais" tanto entre os profissionais de saúde quanto entre a população ${ }^{1}$. O processo de medicalização não está restrito apenas ao momento e ao tipo de parto, mas se estende também aos programas de pré-natal em que se verifica, por exemplo, o alto número de uso de exames de imagem pré-natal. No que se refere, de forma mais pontual, à medicalização do nascimento no país, nas últimas três décadas esta tem sido acompanhada pela persistente alta prevalência de nascimentos prematuros e de mortes maternas e de neonatos 1,2 .

Os avanços das políticas públicas brasileiras de saúde, com a institucionalização do Sistema Único de Saúde (SUS), ampliaram o acesso ao pré-natal 3, porém não com a mesma intensidade a qualidade da atenção 2; com frequência, as ações materno-infantis seguem incorporando concepções restritas e fragmentadas de mulher em sua função maternal 4,5.

Diante desse panorama de alta medicalização, recentemente foram publicadas no Brasil diretrizes visando à reorganização da atenção ao pré-natal e ao parto. Essa é uma política pública eleita como prioritária e que prevê o planejamento reprodutivo e a atenção humanizada à gravidez, ao parto e ao puerpério 6 . Pretende ir ao encontro de evidências recentes que destacam a relevância da atenção primária e das equipes de saúde no que diz respeito ao apoio às mulheres para a escolha do tipo de parto a realizar e para a eleição de métodos de anticoncepção ${ }^{7}$.

A assistência à mulher na gestação e no parto, preconizada pelo SUS, no Brasil, cenário do estudo, propõe a unidade básica de saúde (UBS) como porta de entrada preferencial ao sistema de saúde, ponto de atenção estratégico para o acompanhamento continuado da gravidez. Elege a Estratégia Saúde da Família (ESF) como proposta programática principal para a reorganização dos modelos e práticas materno-infantis brasileiras no âmbito primário de atenção 3,8 .

Porém, entraves de diversas ordens ainda estão presentes na organização do trabalho das equipes de saúde da família no Brasil 9,10. Um conjunto de autores brasileiros apresenta contribuições metodológicas no campo da gestão e do cuidado para a transformação dos serviços de saúde, ainda excessivamente pautados no paradigma biomédico tradicional. Um tema consensual dos estudos refere-se à necessidade de humanizar as práticas de saúde extremamente pautadas pela biomedicina 11,12.

Então, em que medida o processo organizador do cuidado com a mulher gestante adotado pelo sistema público de saúde brasileiro está relacionado com o desfecho entre o parto normal ou a cesárea? A caracterização de um processo de transição do profissional médico responsável, do generalista na atenção primária para o especialista (geralmente obstetra) no cuidado ao alto risco e ao parto no hospital, afeta as escolhas das mulheres? Ou, perguntando de outro modo, como se integram, no percurso das ações de cuidado até o momento do nascimento, por caracterizarem-se como discursos biopolíticos de medicalização \& humanização, as práticas dos médicos generalistas e as dos médicos especialistas?

Colocando em foco a análise dos processos de medicalização \& humanização, não como polaridades dicotômicas do trabalho de equipes de saúde da família na atenção primária, mas constituidores de micropolíticas do trabalho na saúde, questiona-se: como estas disputas biopolíticas estão presentes no cotidiano de atuação das equipes na atenção ao pré-natal no Brasil? Como extremos em enfrentamento ou como componentes do mesmo problema?

O estudo dedica-se a compreender o modo como essas vertentes de análise (medicalização \& humanização), antes de tudo sociológicas, corporificam-se nas realidades de trabalho de equipes na atenção primária à saúde, especialmente no caso da atenção à mulher gestante. Como os profissionais se organizam em nível de equipe para enfrentar problemas e conflitos que podem expressar processos de medicalização \& humanização? E, nesse contexto, de que modo o uso de protocolos e os processos de gestão das competências profissionais vivenciados pelas equipes de saúde são pautados pela medicalização \& humanização? Ainda, como é possível considerar ou avaliar isso?

$\mathrm{Na}$ análise do discurso empreendida com base no material empírico produzido no trabalho de campo, os interesses voltam-se para compreender como os discursos da medicalização \& humanização atravessam e orientam a atuação das equipes na atenção à mulher gestante, suas condições de possibilidade e os efeitos que produzem. 


\section{Medicalização \& humanização, disputas biopolíticas?}

O conceito de medicalização na saúde não é novo. Nos últimos quarenta anos, tem sido alvo de intensa atenção de autores do campo da sociologia da saúde, os quais o interpretam com diferentes argumentos e pontos de vista 13,14,15,16,17,18,19.

Tradicionalmente, estudos críticos à medicalização na saúde valorizam análises das assimetrias de poder presentes nas relações entre a profissão médica e a população leiga. Nessa forma de pensamento, compreende-se a medicalização como "um problema previamente não-médico que passa a ser definido e tratado como médico” 16 (p. 196). São concepções comumente associadas a uma noção unidimensional de medicalização - a dominância médica sobre experiências avaliadas como normais 20. Ao enfatizarem tendências imperialistas da profissão médica subestimando os benefícios da medicina, afastam-se do conceito de biopolítica desenvolvido por Foucault 21, que privilegia analíticas produtivas de poder e não apenas repressivas. No sentido foucaltiano, o (bio)poder exercido sobre a existência humana a transforma em objeto (bio)político para a racionalização da vida coletiva, tornando-se a biopolítica unidade analítica de práticas de intervenção, formas de conhecimento e regimes de autoridade 22 .

A atenção à saúde da mulher na gestação e no parto é um tema que se destaca na arena de debates sobre a medicalização da vida, tendo em vista o elevado número de cesáreas que vem sendo realizado no mundo 23. "Modelos desmedicalizadores" (sociais, naturais, holísticos, humanísticos) de atenção ao pré-natal e ao parto emergem como uma ordem diferente para a prática obstétrica tecnocrática (biomédica) tradicional e baseiam-se no conceito de medicina biopsicossocial 24 .

Porém, processos de assistência à saúde, biomédicos ou holísticos, não são constituídos como oposições binárias; nuances e ambiguidades lhes são inerentes 14,20. A abordagem holística (humanista), ainda que se pretenda desmedicalizadora, ao ampliar a abrangência do processo saúde/doença para quase todos os domínios da vida (psicossocial) é também um impulso na direção da medicalização 14 . As propostas de uma medicina mais humana com a priorização de cuidados psicosociais envolvem extensa regulação da vida social e o uso de tecnologias de controle inerentes aos processos medicalizadores 25. A humanização é uma biopolítica não menos prescritiva que qualquer outra, "mesmo quando explicita a diversidade como valor que deve orientar o processo" 26 (p. 396). O humano será sempre resultado das inter-relações do sujeito com a história, com a cultura e com a sociedade 27 . Lembrando Foucault 21, todo discurso tem como premissa uma agenda e uma dinâmica própria de poder.

Compreende-se que as biopolíticas de humanização \& medicalização na atenção à saúde das mulheres gestantes são práticas sociais que se manifestam como discursos 28 - ou a forma como o discurso se dá e acontece em nível de tecnologia de biopoder. Para Foucault 29 (p. 8) "em toda a sociedade a produção do discurso é simultaneamente controlada, selecionada, organizada e redistribuída por um certo número de procedimentos". Nesse sentido, abordagens sociológicas que privilegiam a análise dos processos de medicalização \& humanização de forma conexa têm sido pouco exploradas e, acreditase, poderiam acionar dimensões extras na compreensão dos temas e problemas próprios desta relação.

\section{Percursos metodológicos}

Foi desenvolvido um estudo de caso do tipo único, integrado, com múltiplas unidades de análise e abordagem qualitativa 30 .

Participaram do trabalho 16 municípios com portes populacionais diversificados $(300.000$ a 3.500 habitantes) em uma macrorregião administrativa de saúde no Estado do Rio Grande do Sul. Os critérios de inclusão das equipes de saúde da família ao estudo foram a adesão, no ano de 2012, ao Programa de Avaliação da Qualidade da Atenção Primária em Saúde (PMAQ) e a presença de equipes de saúde bucal.

Realizou-se 17 grupos focais (com duração de aproximadamente uma hora, conduzidos por um coordenador e dois relatores, filmados e posteriormente transcritos), em 17 UBS do SUS. Um total de 47 trabalhadores (14 médicos, 19 enfermeiros e 14 cirurgiões-dentistas) foram ouvidos.

Realizar grupos focais com as equipes de saúde da família em seus ambientes cotidianos de trabalho foi a estratégia adotada pelo estudo, que objetivou o diálogo em profundidade e com um nível maior de trocas entre os participantes. Os autores procuraram respeitar as discussões, confrontos e 
discordâncias. A intenção foi de ampliar nos dados produzidos a dimensão de interação social 31. A interação dos participantes entre si e com o facilitador conduziu a um contexto multidimensional, permitindo também uma abertura para compreender o modo como macro-políticas sociais poderiam impulsionar as conversas ${ }^{32}$, no caso do objeto em estudo, as biopolíticas de medicalização \& humanização em relação aos processos de trabalho de equipes de saúde. Nesse sentido, pode-se definir o caráter teórico da interação dos participantes no desenho dos grupos focais realizados como de perspectiva social construtivista 31 .

A análise do discurso realizada foi baseada na teorização foucaultiana, visando a compreender como os discursos constitutivos das biopolíticas de medicalização \& humanização atravessam e modulam o que os profissionais dizem "que fazem e a maneira em que ofazem [bem como] as formas de racionalidade que organizam as maneiras de fazer” 33 (p. 337). Nesse sentido, as práticas "vivas” descritas nos grupos focais não são aceitas como representações naturais do mundo social, mas são compreendidas com base nas relações com os discursos que as colocam em funcionamento 34,35. O percurso teórico-metodológico do estudo foi construído para que fosse possível analisar e problematizar como o cuidado de mulheres gestantes realizado pelas equipes de saúde da família é atravessado e, ao mesmo tempo, retroalimenta esses discursos biopolíticos 21 .

O projeto de pesquisa obedece às exigências bioéticas e foi submetido à análise ética pela Plataforma Brasil, tendo recebido parecer consubstanciado aprovado com o número CAAE 16931213.3.0000.5347. Foram considerados participantes da pesquisa apenas os trabalhadores que assinaram o Termo de Consentimento Livre e Esclarecido (TCLE).

\section{Do generalista ao especialista: práticas discursivas no caminho ao nascimento}

A medicina generalista emergiu sob a égide dos princípios da medicina biopsicossocial, dos discursos holísticos e humanistas 36 e da defesa do "modelo" familiar para a atenção à mulher na gestação e no parto 37 . A abordagem humanizada de atenção ao nascimento preconiza que a base de atenção seja em nível comunitário, com profissionais trabalhando em equipes informadas por evidências científicas 38 . Argumenta-se que a interação das famílias com o sistema de saúde, particularmente com os trabalhadores na linha de frente, habilita as mulheres para serem produtoras da própria saúde 39 .

No Brasil, local do estudo, é pluriprofissional o percurso percorrido pela mulher do pré-natal ao parto no SUS. São equipes básicas multiprofissionais (prioritariamente enfermeira, cirurgião-dentista e médico generalista) que realizam o cuidado pré-natal das mulheres na atenção primária. Médicos especialistas (preferencialmente obstetras) se responsabilizam pela realização do parto nos hospitais, em regime de plantão. Adota-se a classificação do risco como critério de elegibilidade para definir a necessidade ou não do encaminhamento da gestante para o acompanhamento pelo obstetra desde o pré-natal 40,41 .

Da forma de pagamento para os procedimentos à percepção do profissional quanto ao desejo da mulher pelo tipo de parto a realizar, passando, dentre outros aspectos, pela utilização de tecnologias médicas ou pelas regulações judiciais da má prática, o saber/fazer obstétrico brasileiro conformou práticas de atenção medicalizadas e com baixa participação de parteiras 42. Parcela menor de mulheres brasileiras possui plano privado de saúde, o que lhes confere, geralmente, a possibilidade de escolher o médico que acompanhará tanto o pré-natal quanto o parto. Mas, para a grande maioria das mulheres que usa o SUS, frequentemente é mais difícil escolher os médicos que irão assisti-las, tanto no prénatal na atenção primária quanto no momento do parto no hospital 41. Recente amplo estudo de base nacional verificou que apenas um quarto das mulheres brasileiras analisadas havia sido assistida pelo mesmo profissional durante o pré-natal e o parto 40.

As problematizações apresentadas até aqui, constituem um fio condutor para a compreensão sobre os modos como discursos constitutivos de biopolíticas de medicalização \& humanização podem estar conformando o trabalho das equipes e da atenção às mulheres gestantes. No estudo realizado, as equipes de saúde nos grupos focais demarcam interposição dos médicos especialistas (ginecologistas e obstetras) na captação e no acompanhamento das mulheres gestantes.

"Eu [médico generalista] estou preparado para atender qualquer pessoa, mas neste posto, existe ginecologista, pediatra, que tem consulta semanal. Os médicos especialistas vêm e atendem, mas se tem alguma 
coisa, algum problema nessas gestantes que tem que ser vista por mim [médico generalista], não há nenhum problema" (E31).

Com muita frequência nos municípios pesquisados, obstetras, ginecologistas e também pediatras atuam igualmente no espaço físico de trabalho dos médicos generalistas das equipes de saúde da família, realizando a assistência à saúde da população que deveria ser de responsabilidade da equipe de atenção básica. $\mathrm{O}$ acesso facilitado ao médico especialista (obstetra e ginecologista) permite que as gestantes, independentemente dos critérios de elegibilidade, optem por buscá-lo espontaneamente e de maneira direta, sem vincular-se ao generalista.

"Como a gente tem o pediatra, que é semanal, e o obstetra, eu [médico generalista] acabo não entrando nessa área, a não ser quando acontece alguma urgência com a gestante ou com o recém-nascido. Daí acabamos fazendo esse acolhimento aqui. Mas o problema de prevenção, do pré-natal e do acompanhamento da criança é feito com os outros profissionais [especialistas]" (E24).

No estudo, essas falas sugerem resistências para a implantação de práticas humanistas, que caracterizam a abordagem generalista preconizada, no caso em questão, pela estratégia de saúde da família. Independentemente de ter sido definida como perspectiva orientadora das políticas públicas brasileiras para a atenção à saúde da mulher, parece que a humanização das práticas não consegue impor-se no confronto cotidiano com a medicina especializada, profundamente incorporada aos princípios do paradigma biomédico tradicional. Mas, de que modo se poderia analisar essas disputas e confrontos, nesse contexto?

Na emergência das proposições generalistas o questionamento do domínio epistemológico biomédico não correspondeu à diminuição da autoridade hospitalar como produtora e detentora da educação médica. O campo de trabalho do generalista expandiu-se em relação às ênfases reconhecidas pela medicina tradicional (por exemplo, obstetrícia, pediatria etc.) e isto também pode ter contribuído para a persistência da hegemonia da formação hospitalar 43. Ênfase que também é evidenciada nas falas dos generalistas da saúde familiar em que, apesar de dizerem que moldam seu fazer orientandose pelas políticas públicas, seguem atribuindo maior legitimidade ao trabalho dos especialistas.

De modo geral, a despeito das fortes recomendações sobre a importância da medicina generalista no acompanhamento ao nascimento, o que se tem verificado é a diminuição constante da participação dos médicos de família nos cuidados obstétricos 44 . Wiegers 45 descreve a opinião de médicos generalistas sobre por que não fazem partos - interferência no estilo de vida, interrupção na rotina, medo de litígios e custos de seguro de malversação, formação insuficiente ou número insuficiente de casos para reter competência. Alguns desses elementos relacionados com a responsabilização pelo cuidado ao pré-natal emergem nas falas das equipes entrevistadas.

"O nosso município parece, pelo menos pelos especialistas, mas também pela própria estratégia de saúde da família, consultório particular. Quem procura é o paciente” (E15).

"Mas, é uma parte que eu [cirurgiã-dentista] estou totalmente alheia, o atendimento de gestantes e recémnascidos (...) não tem vínculo nenhum, não tem uma atenção voltada para isso” (E03).

Em muitas falas o atendimento das gestantes pelos obstetras na atenção primária é entendido como modo de fortalecer a priorização do atendimento médico, a livre demanda e o uso de exames. Essa lógica, no entanto, interfere no estabelecimento do vínculo com as mulheres gestantes e distancia-se dos princípios que pautam o modelo de atenção à saúde da família (integralidade, longitudinalidade e coordenação do cuidado) 46.

O enfrentamento da alta e complexa demanda de necessidades e de prioridades de saúde da população atendida no âmbito da atenção primária justificaria, na opinião dos grupos, a presença dos especialistas. A segurança do apoio técnico oferecido pelo especialista também é defendida por algumas equipes de saúde para a participação e presença dos obstetras no cuidado com as gestantes ainda na atenção primária.

"Quando tem alguma gestante com algum problema ou identifica-se alguma situação mais de risco ou depois mesmo quando os bebês nascem nós fizemos essa visita. Tem essa troca com a ginecologista” (E4).

"Há coisas que tu tens que perguntar para um especialista. Uma mamografia que chega com um resultado que encontrou alguma coisa. Um sangramento importante, uma coisa assim (...) Tu já tens a quem perguntar, já tem quem te dê um apoio (...) É importante sim" (E18).

Na modelação do cuidado no caminho ao nascimento, diferentes níveis de interação dos médicos generalistas com os obstetras são possíveis, da consulta para aconselhamento ou parecer, ao encami- 
nhamento para a transferência completa de cuidados, passando pela colaboração que exigiria a gestão conjunta do caso ${ }^{47}$. É a segunda alternativa a que predomina nessas práticas. Sabe-se que práticas assistenciais colaborativas que incidem na redução de procedimentos tecnológicos (episiotomia e cesárea, por exemplo) são mais frequentes em modelos humanizados de assistência ao nascimento. Assim, a atenção à saúde da mulher gestante caracterizada nas práticas descritas pelos participantes evidencia que as orientações para a preparação das mulheres para o parto não encontram condições de serem priorizadas e que práticas colaborativas são ainda muito pouco usadas nas estratégias de cuidado, atestando o que indicam outros estudos sobre a ainda baixa qualidade da atenção ao prénatal no Brasil 2,41,48.

\section{Do protocolo ao contexto social: governamentalidade da competência profissional}

Práticas políticas, medicina baseada em evidência, guidelines, protocolos, parametrizações - são inúmeras as ferramentas constitutivas do conjunto de instruções dirigidas aos trabalhadores de saúde para direcionar suas ações e tomadas de decisão frente a situações de saúde e doença 49. Mas, a "tecnogovernância” do trabalhador de saúde 50 , valendo-se do conceito de governamentalidade, pressupõe a capacidade de articular aderência simultânea aos protocolos e à medicina centrada na pessoa (ou ao acolhimento da realidade psicossocial). Essa ênfase evidencia que a atualidade exige dos trabalhadores da saúde a difícil tarefa de articular nas práticas de atenção elementos constitutivos dos discursos biomédicos e da humanização. Ou, dito de outro modo, integrar duas visões, dois pólos teoricamente em tensão nas práticas de saúde - medicalização \& humanização.

As relações de poder que modulam a relação profissional de saúde e usuária, na perspectiva foulcautiana, configura-se como uma espécie de link entre as complexas relações que se instalam na cena do encontro clínico. Regulam, mas também serão reguladas, operando por meio de procedimentos disciplinares que lhes serão ditados, tais como, por exemplo, os protocolos clínicos. Na governamentalidade, as práticas profissionais na saúde estão entrelaçadas com as formas de governar e os trabalhadores, tal como especialistas em alianças com autoridades políticas administram o viver minimizando doenças e promovendo saúde individual e coletiva. Nesse jogo de gestão de biopoderes e competências profissionais, os efeitos da medicalização \& humanização serão evidenciados na maneira que o aparato profissional/tecnológico remodela os significados de saúde e de doença e em como afiançam conceitos do que deve ser entendido por humano 15,17,25.

No Brasil, a defesa do discurso de humanização da atenção à saúde foi traduzida como política pública. Os formuladores das políticas brasileiras consideram o sistema de saúde desumano, inadequado e ineficiente, e diagnosticam a necessidade de humanizar o serviço. A política de humanização, inicialmente direcionada à atenção da mulher na gestação e no parto, foi generalizada no país ampliando-se para os serviços de saúde e assumindo destaque no que se entende como prioridade para a superação dos obstáculos da saúde pública brasileira 10. Estudiosos e militantes do tema defendem que a assistência humanizada à mulher deva ser baseada principalmente: na melhor evidência científica, no reconhecimento dos direitos de participação e de autonomia da mulher, na revisão do papel do médico no parto e na relação democrática entre médico/paciente 4.

Nessa direção, reformas humanistas dos sistemas de saúde exigem outras formas de governamento e, por conseguinte, reformas de processos de trabalho, quando o valor das competências dos profissionais emerge nos discursos da gestão dos serviços. Porém, análises sobre competências profissionais ainda estão tradicionalmente centradas na aquisição de conhecimentos e técnicas 51 e/ou no desempenho individual dos trabalhadores 52. Com maior frequência, as competências são tratadas como integração de variáveis dicotômicas: o conhecimento e a prática, o individual e o coletivo, o visível (o trabalho) e o invisível (o emocional), a responsabilidade social e a prática profissional 53. Mais raramente as interrogações voltam-se para as condições dos complexos ambientes de trabalho 54,55 ou para como os trabalhadores recriam competências valendo-se de saberes e valores próprios 56 .

Evidências apontam que, embora os trabalhadores de saúde representem cerca de $10 \%$ da força de trabalho brasileira, vários desafios permanecem, tais como, distribuição regional desigual de pessoal qualificado, alta rotatividade, escassez de carreiras estruturadas e grandes diferenças salariais 
entre regiões, estados, municípios e equipe ${ }^{57}$. As práticas observadas neste estudo apresentam-se particularmente afetadas pelas incertezas do trabalho: a alta rotatividade, a precariedade de vínculos empregatícios e a sobrecarga de trabalho (principalmente das enfermeiras que acumulam a função de gestão e atenção nas equipes). A incorporação de princípios e pressupostos da humanização fica prejudicada na gestão das competências profissionais, destacando-se aspectos como as possibilidades de inovações nos processos de trabalho e o trabalho em equipe.

O impulso contemporâneo de regulação e auditoria das práticas de saúde que exorta médicos (e profissionais de saúde) a aderirem às diretrizes e protocolos baseados em evidências para guiar as práticas clínicas, pressupõe aumentar a eficiência por meio da reorganização e racionalização do trabalho. Um discurso que afasta o interesse da atenção centrada no usuário, próprio das abordagens humanistas, em favor de uma retórica de implementar referenciamentos em nome da produtividade. Valores de gestão que terminam por incentivar a medicina generalista a situar-se no espaço biomédico $58,59,60$.

Nas práticas observadas no estudo, o protocolo recomendado pelo SUS, que preconiza que o acompanhamento pré-natal da mulher gestante deva ser realizado pelo generalista (seguindo modelos humanizados) não é cumprido. Pelo protocolo o obstetra acompanharia apenas as gestantes de alto risco, ou seja, as que possuam agravos que possam colocar em risco a saúde da mulher ou da criança. Entretanto, também as gestantes de baixo risco são acompanhadas por obstetras.

"O município não tem protocolo, no caso de gestantes o atendimento é feito pelo ginecologista e dos bebês pelo pediatra e não pelo médico [generalista] da saúde da família” (E28).

"Mas, acompanhar o pré-natal é o obstetra que faz, (...) apesar de estar escrito direitinho no programa de saúde da família que tem que acompanhar as gestantes. Eles [os médicos generalistas] dizem que a gestante não é deles e que a gente tem que ter o especialista para cuidar (E42)".

Assim, o fazer das equipes de saúde situa-se no caminho do meio entre a coordenação do cuidado e a posição de coadjuvantes dos obstetras. As equipes se responsabilizam apenas pelos protocolos de atendimento das urgências das mulheres gestantes no território. Muitos dos cuidados básicos de acompanhamento clínico que deveriam ser de responsabilidade do médico generalista na atenção primária serão realizados pelos obstetras.

"Ele [médico generalista] só atende intercorrências com as gestantes. (...) A obstetra está uma vez por semana aqui. Ela tem doze horas para duas unidades de saúde. Então as intercorrências o médico [generalista] da saúde da família faz, ou um primeiro atendimento" (E42).

Em tempos de avaliação de sistemas e serviços de saúde que valorizam indicadores, é preciso refletir sobre as propriedades inerentes ao trabalho na saúde, em que a complexidade do objeto e a baixa maleabilidade espacial e temporal devido à exigência de copresença limitam as possibilidades de racionalização e (re)organização do trabalho na saúde 61 .

Um desafio extra para a gestão da "tecnogovernância" das competências profissionais nas novas dinâmicas de organização em rede e padrão policêntrico de poder é a autonomia inerente aos trabalhadores que compõem os sistemas, e a educação passa a assumir também um papel de regulação dos conflitos e interesses 62 . No entanto, no contexto pesquisado as possibilidades de acesso a processos de qualificação são escassas, assim como há dificuldades na manutenção dos espaços de reuniões de equipes. A aproximação entre a gestão, a educação permanente e as práticas clínicas é ainda um caminho a ser percorrido, mas no sentido da problematização sobre os processos de medicalização \& humanização como constituidores das próprias práticas de atenção.

\section{Considerações finais}

As práticas de atenção às mulheres gestantes analisadas pelo estudo indicam que a abordagem humanizada, ênfase principal das responsabilidades das equipes de saúde da família, está em desvantagem na relação com as práticas medicalizadas demandandas dos médicos especialistas (obstetras). Porém, a peculiaridade no estudo reside justamente no entendimento de que as equipes de saúde da família não atuam em oposição ao modelo biomédico, pelo contrário, possibilitam e fortalecem a demanda pelo obstetra. Assim, é possível dizer que encontram-se mais pontos em conexão nas biopolíticas de medicalização \& humanização do que propriamente em disputa. 
No caso do modelo brasileiro aqui apresentado, os resultados apontam para o problema de interlocução entre equipes de saúde generalistas e obstetras. No contexto estudado, a ênfase na atenção humanizada à mulher na gestação e no parto poderia ampliar o caráter biopsicossocial do cuidado, interferindo nos desenhos e fronteiras dos territórios profissionais e demandando renegociação de competências das equipes e trabalhadores envolvidos. Mas, verifica-se que esforços de colaboração empreendidos entre as equipes de saúde da família e os obstetras ainda não são suficientes para redefinir essas relações. As políticas de governamentalidade das competências profissionais na atenção ao pré-natal nos municípios estudados não investem no desenvolvimento dessas relações interprofissionais. Os cuidados realizados com as mulheres pelas equipes de saúde generalistas durante a gestação ainda estão muito afastados daqueles realizados pelos obstetras, como se fossem projetos profissionais com objetivos distintos.

Nessa direção, o estudo permite concluir que as práticas das equipes de saúde generalistas são legitimadas pelos gestores das políticas públicas, mas a responsabilidade profissional não se afasta do escopo de ação dos obstetras. As equipes de saúde da família referem dificuldades para realizar o cuidado pré-natal de mulheres gestantes que lhe é atribuído. Entretanto, não se trata aqui somente da presença do obstetra; a prevalência de um conjunto de elementos que definam as competências e o agir profissional com as mulheres gestantes seguem fragmentando as práticas de cuidado dos médicos generalistas, centrado-as nos obstetras.

\section{Colaboradores}

C. M. Warmling desenvolveu a concepção do projeto, coleta, análise e interpretação dos resultados, redação e revisão do artigo. A. P. Fajardo e D. E. Meyer contribuíram na revisão crítica e redação do artigo. C. F. Bedos contribuiu na concepção, redação e revisão do artigo.

\section{Agradecimentos}

Agradecemos à especialista em saúde do Governo do Estado do Rio Grande do Sul Janice Castilhos Gomes, pelo apoio na realização dos grupos focais nos municípios. $\mathrm{O}$ artigo foi produzido durante a concessão de bolsas de estudos pela Cooperação Internacional Programa Capes/Cofecub na McGill University, financiada pela Capes, Ministério da Educação, e pelo CNPq, FAPERGS/MS/CNPq/ SESRS.

\section{Referências}

1. Declercq E. Childbirth in Brazil: challenging an interventionist paradigm. Birth 2015; 42:1-4.

2. Victora CG, Aquino EML, Carmo Leal M, Monteiro CA, Barros FC, Szwarcwald CL. Maternal and child health in Brazil: progress and challenges. Lancet 2011; 377:1863-76.

3. Paim J, Travassos C, Almeida C, Bahia L, Macinko J. The Brazilian health system: history, advances, and challenges. Lancet 2011; 377:1778-97.

4. Diniz S. Materno-infantilism, feminism and maternal health policy in Brazil. Reprod Health Matters 2012; 20:125-32.

5. Klein C, Meyer DE, Borges ZN. Políticas de inclusão social no Brasil contemporâneo e educação da maternidade. Cad Pesqui 2013; 43:906-23.

6. Ministério da Saúde. Portaria no 1.459 , de 24 de junho de 2011. Institui no âmbito do Sistema Único de Saúde SUS - a Rede Cegonha. Diário Oficial da União 2011; 27 jun.

7. Brocklehurst P, Hardy P, Hollowell J, Linsell L, Macfarlane A, Marlow N, et al. Perinatal and maternal outcomes by planned place of birth for healthy women with low risk pregnancies: the Birthplace in England national prospective cohort study. BMJ 2011; 343:d7400.

8. Macinko J, Harris MJ. Brazil's Family Health Strategy - delivering community-based primary care in a universal health system. $\mathrm{N}$ Engl J Med 2015; 372:2177-81.

9. Trad LAB, Rocha AARM. Condições e processo de trabalho no cotidiano do Programa Saúde da Família: coerência com princípios da humanização em saúde. Ciênc Saúde Coletiva 2011; 16:1969-80. 
10. Nora CRD, Junges JR. Política de humanização na atenção básica: revisão sistemática. Rev Saúde Pública 2013; 47:1186-200.

11. Campos GWS. O anti-Taylor: sobre a invenção de um método para co-governar instituições de saúde produzindo liberdade e compromisso. Cad Saúde Pública 1998; 14:863-70.

12. Ayres JRDCM. Organização das ações de atenção à saúde: modelos e práticas. Saúde Soc 2009; 18 Suppl 2:11-23.

13. Zola IK. Medicine as an institution of social control. Sociol Rev 1972; 20:487-504.

14. Lowenberg JS, Davis F. Beyond medicalisation-demedicalisation: the case of holistic health. Sociol Health Illn 1994; 16:579-99.

15. Lupton D. Foucault and medicalisation critique. In: Petersen A, Buntton R, editors. Foucault, health and medicine. New York/London: Routledge; 1997. p. 94-110.

16. Conrad P. Medicalization: changing contours, characteristics, and contexts. In. Cockerham WC, editor. Medical sociology on the move new directions in theory. New York: Springer; 2013. p. 195-214.

17. Rose N. Beyond medicalisation. Lancet 2007; 369:700-2.

18. Halfmann D. Recognizing medicalization and demedicalization: discourses, practices, and identities. Health (London) 2012; 16:186-207.

19. Bell SE, Figert AE. Medicalization and pharmaceuticalization at the intersections: looking backward, sideways and forward. Soc Sci Med 2012; 75:775-83.

20. Ballard K, Elston MA. Medicalisation: a multidimensional concept. Soc Theory Health 2005; 3:228-41.

21. Foucault M. Nacimiento de la biopolítica: curso del Collège de France (1978-1979). Buenos Aires: Fondo de Cultura Económica; 2007.

22. Rabinow P, Rose N. Biopower today. Biosocieties 2006; 1:195-217.

23. Goldenberg RL, McClure EM, Bhutta ZA, Belizán JM, Reddy UM, Rubens CE, et al. Stillbirths: the vision for 2020. Lancet 2011; 377:1798-805.

24. Goer H. Humanizing birth: a global grassroots movement. Birth 2004; 31:308-14.

25. Nettleton S. The sociology of health and illness. 2nd Ed. Cambridge: Polity Press; 2006.

26. Gastaldo D. Humanização como processo conflitivo, coletivo e contextual. Interface Comun Saúde Educ 2005; 9:395-7.

27. Meyer DE. Como conciliar humanização e tecnologia na formação de enfermeiras/os? Rev Bras Enferm 2002; 55:189-95.

28. Oakley A, Hickey D, Rajan L, Rigby AS. Social support in pregnancy: does it have long-term effects? J Reprod Infant Psychol 1996; 14:7-22.

29. Foucault M. A ordem do discurso: aula inaugural no Collège de France, pronunciada em 2 de dezembro de 1970. 11a Ed. São Paulo: Edições Loyola; 2004.

30. Yin RK. Estudo de caso: planejamento e métodos. Porto Alegre: Bookman; 2010.
31. Ryan KE, Gandha T, Culbertson MJ, Carlson C. Focus group evidence: implications for design and analysis. Am J Eval 2014; 35:328-45.

32. Hollander J, Gordon H. The processes of social construction in talk. Symb Interact 2006; 29:183-212.

33. Castro E. Vocabulário de Foucault: um percurso pelos seus temas, conceitos e autores. Belo Horizonte: Autêntica Editora; 2009.

34. Fischer RMB. Foucault e a análise do discurso em educação. Cad Pesqui 2001; (114):197-223.

35. Traversini CS, Ferreira MS. A análise Foucaultiana do discurso como ferramenta metodológica de pesquisa. Educação \& Realidade 2008; 38:207-26.

36. Hasegawa H, Reilly D, Mercer SW, Bikker A. Holism in primary care: the views of Scotland's general practitioners. Prim Health Care Res Dev 2005; 6:320-8.

37. Jimenez V, Klein MC, Hivon M, Mason C. A mirage of change: family-centered maternity care in practice. Birth 2010; 37:160-7.

38. Wagner M. Fish can't see water: the need to humanize birth. Int J Gynecol Obstet 2001; 75 Suppl 1:25-37.

39. Darmstadt GL. Stillbirths: missing from the family and from family health. Lancet 2011; 377:1550-1.

40. Domingues RMSM, Dias MAB, NakamuraPereira M, Torres JA, d'Orsi E, Pereira APE, et al. Processo de decisão pelo tipo de parto no Brasil: da preferência inicial das mulheres à via de parto final. Cad Saúde Pública 2014; 30 Suppl 1:S101-16.

41. Viellas EF, Domingues RMSM, Dias MAB, Gama SGN, Teme Filha MM, Costa JV, et al. Assistência pré-natal no Brasil. Cad Saúde Pública 2014; 30 Suppl 1:S85-100.

42. Patah LEM, Malik AM. Modelos de assistência ao parto e taxa de cesárea em diferentes países. Rev Saúde Pública 2011; 45:185-94.

43. Armstrong D. Theoretical tensions in biopsychosocial medicine. Soc Sci Med 1987; 25:1213-8.

44. Rayburn WF, Petterson SM, Phillips RL. Trends in family physicians performing deliveries, 2003-2010. Birth 2014; 41:26-32.

45. Wiegers TA. General practitioners and their role in maternity care. Health Policy 2003; 66:51-9.

46. Starfield B. Atenção primária: equilíbrio entre necessidades de saúde, serviços e tecnologia. Brasília: Organização das Nações Unidas para a Educação, a Ciência e a Cultura/Ministério da Saúde; 2002.

47. Skinner JP, Foureur M. Consultation, referral, and collaboration between midwives and obstetricians: lessons from New Zealand. J Midwifery Womens Health 2010; 55:28-37.

48. Vogt SE, Silva KS, Dias MAB. Comparison of childbirth care models in public hospitals, Brazil. Rev Saúde Pública 2014; 48:304-13.

49. Berg M. Problems and promises of the protocol. Soc Sci Med 1997; 44:1081-8. 
50. May C, Rapley T, Moreira T, Finch T, Heaven B. Technogovernance: evidence, subjectivity, and the clinical encounter in primary care medicine. Soc Sci Med 2006; 62:1022-30.

51. Fullerton JT, Ghérissi A, Johnson PG, Thompson JB. Competence and competency: core concepts for international midwifery practice. Int J Childbirth 2011; 1:4-12.

52. Boreham N. A theory of collective competence: challenging the neo-liberal individualism of performance at work. British Journal of Educational Studies 2004; 52:5-17.

53. Fernandez N, Dory V, Ste-Marie LG, Chaput M, Charlin B, Boucher A. Varying conceptions of competence: An analysis of how health sciences educators define competence. Med Educ 2012; 46:357-65.

54. Hampson I, Junor A. Putting the process back in: rethinking service sector skill. Work Employ Soc 2010; 24:526-45.

55. Armstrong P. Puzzling skills: feminist political economy approaches. Can Rev Sociol 2013; 50:256-83.

56. Schwartz Y. Uso de si e competência. In: Schwartz Y, Durrive L, organizadores. Trabalho \& ergologia: conversas sobre a atividade humana. Niterói: Eduff; 2007.
57. Victora CG, Barreto ML, Carmo Leal M, Monteiro CA, Schmidt MI, Paim J, et al. Health conditions and health-policy innovations in Brazil: the way forward. Lancet 2011; 377:2042-53.

58. Nettleton S, Burrows R, Watt I. How do you feel doctor? An analysis of emotional aspects of routine professional medical work. Soc Theory Health 2008; 6:18-36.

59. Harrison S. New labour, modernisation and the medical labour process. J Soc Policy 2002; 31:465-85.

60. Nettleton S, Burrows R, Watt I. Regulating medical bodies? The consequences of the "modernisation" of the NHS and the disembodiment of clinical knowledge. Sociol Health Illn 2008; 30:333-48.

61. Cohen RL. Time, space and touch at work: body work and labour process (re) organisation. Sociol Health Illn 2011; 33:189-205.

62. Weber SM. The intrapreneur and the mother: strategies of fostering and developing the entrepreneur of the self in organizational development and affirmative action. In: Peters MA, Besley T, editors. Why Foucault? New directions in educational research. New York: Peter Lang; 2007; p. 101-24. 


\section{Abstract}

The study's main objective is to analyze how discourses of medicalization and humanization reconnect in primary healthcare and shape prenatal care for pregnant women provided by family health teams. This was a single and integrated case study with multiple analytical units and a qualitative approach. A total of 17 focus groups were performed, in which 47 health professionals were heard (14 physicians, 19 nurses, and 14 dentists), members of 17 family health teams in 16 municipalities in the South of Brazil. The empirical material was analyzed from the perspective of Foucauldian discourse analysis. The family health teams, adopting general practice, reported difficulties in conducting prenatal care, evoking and bolstering the discourse of obstetric medicalization that their practice should supposedly offset. The discourse officially adopted by humanization, prioritized in the generalist model of prenatal care, continues to function as a complementary discourse to that of medicalization and specialization, which prevails in the practices reported by the teams. The emphasis on humanized care for pregnant women tests the limits of professional territories and assumes the renegotiation of competencies. Efforts at collaboration between the family health teams and obstetricians have not proved very successful in this specific case.

Medicalization; Humanizing Delivery; Pregnant Women

\section{Resumen}

El objetivo principal de este trabajo es analizar cómo los discursos de medicalización y humanización se vuelven a articular entorno a la atención primaria en salud y conforman el cuidado prenatal de mujeres embarazadas, realizado a través de equipos de salud orientados a la familia. Se trata de un estudio de caso de tipo único e integrado, con múltiples unidades de análisis y enfoque cualitativo. Se formaron 17 grupos focales y se escucharon a 47 trabajadores (14 médicos, 19 enfermeros y 14 cirujanos dentales), que componian 17 equipos de salud orientados a la familia en 16 municipios en el sur de Brasil. El material empírico se analizó desde la perspectiva del análisis del discurso foucaultiano. Los equipos de salud orientados a la familia, profesionales de medicina general, informaron sobre las dificultades para realizar el cuidado prenatal de las mujeres gestantes, evocando $y$ fortaleciendo el discurso de la medicalización obstétrica, cuya práctica debería estar debilitándose. El discurso oficialmente adoptado en pro de la humanización, privilegiado en el modelo generalista de atención a las mujeres gestantes, sigue funcionando como discurso complementario al de la medicalización y de la especialización, que prevalece en las prácticas relatadas. El énfasis en la atención humanizada a la mujer en la gestación interfiere en las fronteras de las áreas profesionales y presupone la renegociación de competencias. Los esfuerzos de colaboración emprendidos entre los equipos de salud orientados a la familia y los obstetras no presentan mucho éxito.

Medicalización; Parto Humanizado; Mujeres

Embarazadas
Recebido em 21/Jan/2017

Versão final reapresentada em 14/Set/2017

Aprovado em 31/Out/2017 